\title{
Control de la interferencia en el trastorno por déficit de atención e hiperactividad (TDAH): revisión*
}

\author{
Interference Control in Attention Deficit and Hyperactivity Disorder \\ (ADHD): Review
}
Giomar Jiménez-Figueroa ${ }^{1} \underline{\mathrm{ORCID}}$, José Armando Vidarte Claros $^{2} \underline{\mathrm{ORCID}}$, Francia Restrepo de Mejía ${ }^{3} \underline{O R C I D}$
${ }^{1}$ Universidad Simón Bolívar

${ }^{2} 3$ Universidad Autónoma de Manizales (UAM)

Colombia

Fecha correspondencia:

Recibido: noviembre 25 de 2018.

Aceptado: octubre 31 de 2019.

Forma de citar:

Jiménez-Figueroa, G., Vidarte

Claros, J. A., \& Restrepo de Mejía, F.

(2020). Control de la interferencia

en el trastorno por déficit de atención e hiperactividad (TDAH): revisión. Rev. CES Psico, 13(1), 104-124.

\section{Open access \\ (c) Copyright \\ Licencia creative commons \\ Etica de publicaciones \\ Revisión por pares \\ Gestión por Open Journal System \\ DOl: http://dx.doi.org/10.21615/ \\ cesp.13.1.7 \\ ISSN: 2011-3080}

Sobre el artículo:

* Producto derivado de la tesis de investigación "Control de la interferencia y reconocimiento emocional en el TDAH" del doctorado

Comparte

A

\section{Resumen}

Introducción: Investigaciones sobre el trastorno por déficit de atención e hiperactividad (TDAH) han evidenciado alteraciones en los procesos inhibitorios, en especial en el control de la interferencia, lo que explicaría las dificultades de los niños con TDAH para inhibir estímulos distractores y controlar su comportamiento. Objetivo: Identificar la literatura científica disponible sobre el desempeño en tareas que evalúan el control de la interferencia en niños con TDAH. Método: Se realizó una búsqueda de artículos científicos en las bases de datos de Pubmed, Scopus, Web of Sciencie con los términos ADHD (Attention Deficit Hyperactivity Disorder), Interference Control, Stroop y Flanker, publicados entre los años 2010 y 2017. Se identificaron 520 artículos, después de aplicar los criterios de inclusión y exclusión se seleccionaron 33 para hacer el análisis descriptivo. Resultados: El control de la interferencia se ve afectado en niños con TDAH, evidenciado en mayores tiempos de reacción, de ejecución, variabilidad de respuesta y porcentaje de errores, principalmente en los ensayos incongruentes. Igualmente, se mostraron patrones de activación cortical alterados y variaciones en potenciales evocados cognitivos. Conclusiones: El control de la interferencia se encuentra alterado en niños con TDAH, y el uso de más de un método de evaluación de los niños con este Trastorno puede ser útil para brindar más información del funcionamiento en el control de la interferencia en términos de tiempo y precisión en las tareas.

Palabras claves: Control de la Interferencia. Niños. Funciones Ejecutivas. TDAH. Stroop. Flanker.

\section{Introduction}

Research on attention deficit hyperactivity disorder (ADHD) has shown alterations in interference control, which would explain the difficulties of children with $A D H D$ to inhibit distracting stimuli and regulate their behavior. Objective: identifying available scientific literature about performance on task that evaluate the interference control in children with ADHD. Method: a search on Pubmed, Scopus and Web of Science databases with the terms 
en Ciencias Cognitivas de la Universidad Autónoma de Manizales. Agradecimiento a Colciencias, convocatoria 673 de 2014. Las instituciones patrocinadoras del estudio no tuvieron ningún papel en el diseño del estudio, la recolección de datos, el análisis de datos, la interpretación de datos o la redacción del manuscrito.

\section{Sobre los autores:}

1. Estudiante de Doctorado en Ciencias Cognitivas de la UAM. Magister en Neuropsicología. Psicóloga. Investigadora Grupo Neurociencias del Caribe, Unidad de Neurociencias Cognitivas, Universidad Simón Bolívar, Barranquilla-Colombia.

\section{PhD. en Ciencias de la} Actividad Física y el Deporte. Profesor Titular UAM. Líder del Grupo de Investigación Cuerpo -Movimiento, UAM, ManizalesColombia.

3. Doctora en Ciencias Sociales, Niñez y Juventud. Médica cirujana, Especialista en Medicina Física y Rehabilitación. Especialista en Desarrollo Educativo y Social. Directora del Laboratorio de Neurofisiología de la UAM, Manizales-Colombia.
ADHD, interference control, Stroop and Flanker, published between 2010 and 2017 was performed. 520 articles were identified, after applying the inclusion and exclusion criteria 33 articles were selected for descriptive analysis. Results: interference control is affected in children with ADHD, evidenced in greater reaction and execution times, variability of response and a high errors rate, primarily in incongruent trials. Altered cortical activation patterns and variations in cognitive evoked potentials were also shown. Conclusions: Interference control is altered in children with ADHD, the use of more than one evaluation method may be useful to provide more information on the functioning of interference control in terms of time and accuracy on tasks.

Keywords: Interference Control. Children. Executive Functions. ADHD. Stroop. Flanker.

\section{Introducción}

El trastorno por déficit de atención e hiperactividad (TDAH) es una condición del neurodesarrollo que genera síntomas de inatención y de hiperactividad-impulsividad, los cuales son más frecuentes que los esperados para la edad y el contexto social. La prevalencia del TDAH reportada en el DSM-V es de $5 \%$ en niños y $2,5 \%$ en adultos (American Psychiatric Association, 2013); en el ámbito internacional se han encontrado prevalencias de 8,7\% y 10,6\% en Estados Unidos (Wolraich et al., 2014), 3\% en Italia (Bianchini et al., 2013), 9,11\% y 14,6\% en México (Cornejo-Escatell, Fajardo-Fregoso, López-Velázquez, Soto-Vargas, \& Ceja-Moreno, 2015; Cruz et al., 2010); y en el nacional, en Colombia, se han presentado prevalencias de 5,7\% en la ciudad de Bogotá (Vélez, Talero, González, \& Ibáñez, 2008), de 8.2\% y 7.1\% en Manizales (Pineda et al., 2001), 15\% en Barranquilla (Llanos, García, González, \& Puentes-Rozo, 2019), 15,86\% en Sabaneta-Antioquia (Cornejo et al., 2005), y 16\% en la ciudad de Cali (Bará-Jiménez. Vicuña, Pineda, \& Henao, 2003).

Investigaciones sobre el TDAH han evidenciado la existencia de una alteración en la Función Ejecutiva (FE), en especial en la inhibición de la respuesta tanto cognitiva como comportamental, lo que explicaría las dificultades de estos niños para detener una conducta impulsiva en curso, controlar la interferencia durante tareas que requieren un alto nivel de concentración, organizar las tareas y/o actividades a realizar y coordinar/monitorear sus conductas (Barkley, 1997). Este déficit en la inhibición de la respuesta ha suscitado diversos estudios en función de establecer diferencias significativas para intentar establecer los endofenotipos del TDAH familiar, que permitan detectar genéticamente los subtipos de TDAH o identificar el riesgo genético del Trastorno (Crosbie, Perusse, Barr, \& Schachar, 2008; Goos, Crosbie, Payne, \& Schachar, 2009; Schachar et al., 2005).

Otras investigaciones se han dirigido a identificar los procesos neurocognitivos responsables de los comportamientos de inatención, hiperactividad e impulsividad en niños con TDAH (Gómez-Betancur, Pineda, \& Aguirre-Acevedo, 2005; Pineda, Ardila, \& Rosselli, 1999; Pineda et al., 1998; Trujillo-Orrego, Ibanez, \& Pineda, 2012), y han señalado a las FE, en especial el control inhibitorio, como fuente principal de estos problemas comportamentales (Oosterlaan, Logan, \& Sergeant, 1998; Puentes-Rozo et al., 2015; Willcutt, Doyle, Nigg, Faraone, \& Pennington, 2005).

La terminología relacionada al control inhibitorio varía entre autores y modelos teóricos, sin embargo, se ha mantenido una clara distinción entre la habilidad para prevenir o suprimir una respuesta altamente automatizada o dominante y la habilidad para filtrar información competitiva que es irrelevante a la tarea que se está 
Pág 106

El tiempo de reacción, entendiéndose como el tiempo que pasa entre la presentación de un estímulo y la respuesta del sujeto, se convierte en un aspecto principal en la evaluación del control de la interferencia y de los procesos inhibitorios en general, y varios autores lo han planteado como un componente endofenotípico del TDAH (Crosbie et al., 2008; Goos et al., 2009; Jimenez-Figueroa et al., 2017; Schachar et al., 2005). realizando. La primera ha sido referida como inhibición de respuesta prepotente/ dominante (Barkley, 1997; Friedman \& Miyake, 2004) o supresión de respuesta (Nigg, 2000), mientras que la segunda ha sido denominada como resistencia al factor de interferencia (Friedman \& Miyake, 2004), resolución de conflicto (Posner \& DiGirolamo, 1998) o control de la interferencia (Nigg, 2000).

Existe evidencia que indica que los sujetos con TDAH presentan dificultades en tareas e inhibición de respuestas (Alderson, Rapport, \& Kofler, 2007; Lijffijt, Kenemans, Verbaten, \& van Engeland, 2005; Oosterlaan et al., 1998), pero cuando se refiere al control de la interferencia los hallazgos son menos claros si se tiene en cuenta los tiempos de reacción (van Mourik et al., 2009). En este sentido, el tiempo de reacción, entendiéndose como el tiempo que pasa entre la presentación de un estímulo y la respuesta del sujeto, se convierte en un aspecto principal en la evaluación del control de la interferencia y de los procesos inhibitorios en general, y varios autores lo han planteado como un componente endofenotípico del TDAH (Crosbie et al., 2008; Goos et al., 2009; Jimenez-Figueroa et al., 2017; Schachar et al., 2005).

La investigación sobre el control de la interferencia en el TDAH se ha realizado principalmente a través de tareas tipo Stroop. Esta prueba presenta variantes, aunque usualmente consta de tres fases: fase de lectura, durante la que el participante debe leer una lista de nombres de colores; fase de denominación/color, durante la que se debe nombrar el color presentado en un conjunto de letras "X" (p.e. XXXXX en color azul); y fase conflicto, en la que el sujeto debe nombrar el color de la tinta utilizada y evitar la lectura de la palabra (p. e. la palabra rojo escrita en color azul) (Golden, 1994; Homack \& Riccio, 2004; Mullane, Corkum, Klein, \& McLaughlin, 2009).

Diversos metaanálisis sobre la utilización de tareas tipo Stroop, han evidenciado variaciones en el desempeño de los niños con TDAH y los de los grupos controles, con tamaños del efecto de 0,69 (Mullane et al., 2009; Pennington \& Ozonoff, 1996), 0,75 (Homack \& Riccio, 2004; Mullane et al., 2009) y 0,35 (Mullane et al., 2009; Van Mourik, Oosterlaan, \& Sergeant, 2005). Lansbergen, Kenemans, y van Engeland (2007) realizaron un análisis de las diferentes versiones de calificación de la prueba Stroop, y encontraron que aquellos estudios en los que se utilizó una prueba computarizada del Stroop hallaron mayores diferencias intragrupales al tener en cuenta los tiempos de reacción en cada una de las fases. Aunque las tareas tipo Stroop se utilizan con mucha frecuencia para la evaluación del control de la interferencia, algunos autores han encontrado una limitación en este tipo de tareas relacionada con la presencia de otras variables como la habilidad y velocidad en la lectura, las cuales pueden influir en el desempeño de los niños con TDAH (Van Mourik et al., 2005).

En consecuencia, se han desarrollado otros paradigmas como la tarea tipo Flanker (o de flancos) para evaluar el control de la interferencia sin incluir el componente de lectura. En este tipo de tarea, el participante debe responder a un estímulo objetivo o diana, el cual se encuentra rodeado o flanqueado por dos estímulos intervinientes o distractores a su derecha y a su izquierda. Por lo general, se presentan cinco estímulos (p. e. flechas o peces) apuntando hacia una dirección (derecha o izquierda) y el sujeto debe indicar la dirección del estímulo central ignorando los demás estímulos que le rodean, las cuales pueden apuntar hacia una misma dirección (condición congruente; p. e. $\rightarrow \rightarrow \rightarrow \rightarrow \rightarrow$ ), en dirección contraria (condición incongruente; p. e. $\rightarrow \rightarrow \leftarrow \rightarrow \rightarrow$ ) o en condiciones neutrales $(==\rightarrow==)$ (Homack \& Riccio, 2004; Lansbergen et al., 2007). Y las tareas tipo Simon, en las que el estímulo [p.e. flecha] se presenta a la derecha o izquierda de la pantalla y el sujeto debe indicar la posición del estímulo ignorando la 
Pág 107

De forma general, se ha observado que los niños con TDAH pueden tener un buen resultado en las diferentes tareas, pero con tendencia a presentar tiempos de reacción más largos con alta variabilidad en sus respuestas (Fall, Querne, Le Moing, \& Berquin, 2015; Ma et al., 2018; van Mourik, Sergeant, Heslenfeld, Konig, \& Oosterlaan, 2011), lo que podría sugerir que el tiempo adicional que se toman los niños con TDAH en realizar la tarea sería una acción compensatoria de su dificultad para ignorar eficazmente estímulos irrelevantes y poder finalizar de manera adecuada las actividades. dirección de éste). Mullane et al. (2009) realizaron un metaanálisis del control de la interferencia evaluado con tareas tipo Flanker y Simon, evidenciando que los niños con TDAH presentan desventajas específicas en términos de tiempo de reacción, porcentaje de errores y eficiencia en el desempeño en los ensayos incongruentes con relación a los ensayos congruentes.

De forma general, se ha observado que los niños con TDAH pueden tener un buen resultado en las diferentes tareas, pero con tendencia a presentar tiempos de reacción más largos con alta variabilidad en sus respuestas (Fall, Querne, Le Moing, \& Berquin, 2015; Ma et al., 2018; van Mourik, Sergeant, Heslenfeld, Konig, \& Oosterlaan, 2011), lo que podría sugerir que el tiempo adicional que se toman los niños con TDAH en realizar la tarea sería una acción compensatoria de su dificultad para ignorar eficazmente estímulos irrelevantes y poder finalizar de manera adecuada las actividades.

Esta revisión se realiza con el objetivo de identificar la literatura científica disponible sobre el desempeño en tareas que evalúan el control de la interferencia en niños con TDAH, con el propósito de contribuir al conocimiento sobre la manera en que se presenta esta habilidad en dicha población, teniendo en cuenta sus fortalezas y debilidades, con el fin de proporcionar elementos que favorezcan el diseño e implementación de actividades y programas dirigidos a apoyar y fortalecer su desempeño académico, principalmente.

\section{Método}

Se realizó una búsqueda de literatura para localizar estudios publicados en revistas científicas especializadas sobre control de la interferencia que utilizaran una tarea tipo Flanker y/o Stroop con una muestra de niños con TDAH y un grupo control o con desarrollo típico, teniendo en cuenta los lineamientos generales de búsqueda y filtrado sugeridos por el modelo PRISMA (Moher, Liberati, Tetzlaff, Altman, \& Prisma Group, 2009). Se buscaron en las bases de datos, Medline, Scopus y Web of Science publicados entre los años 2010-2017, los términos utilizados fueron: ADHD (Attention Deficit Hyperactivity Disorder), interference control OR Stroop OR Flanker, child OR boys OR girls, los cuales debían aparecer en el título, resumen o palabras claves. Como criterios de selección, se aceptaron únicamente estudios empíricos con un grupo con diagnóstico confirmado de TDAH, que las edades de la muestra se encontraran entre los 6 - 17 años, que utilizaron el test de Stroop en cualquiera de las dos versiones (papel o computarizado) manteniendo el principio básico de interferencia (palabra-color), y para los estudios que incluían además un grupo con diagnóstico diferente al TDAH, se aceptaron aquellos cuyos análisis se realizaron por grupos separados y, por último, se descartaron los estudios que reportaron datos de tratamiento farmacológico.

La Figura 1 muestra el diagrama de flujo de los procesos de selección y filtrado. En la fase de Identificación, se encontraron 520 artículos científicos. En la fase de rastreo, los artículos duplicados fueron excluidos. Así, el número de artículos disminuyó a 252. Después de aplicar los criterios de inclusión y exclusión a los resúmenes, se mantuvieron 80 artículos. En la fase de elegibilidad, cada artículo con texto completo fue clasificado nuevamente usando los criterios de inclusión y exclusión. De este último filtrado, 33 artículos fueron seleccionados y los datos de los artículos fueron extraídos tomando como guía el modelo de extracción de datos de Cochrane (Ryan. Synnot, Prictor, \& Hill, 2016). 


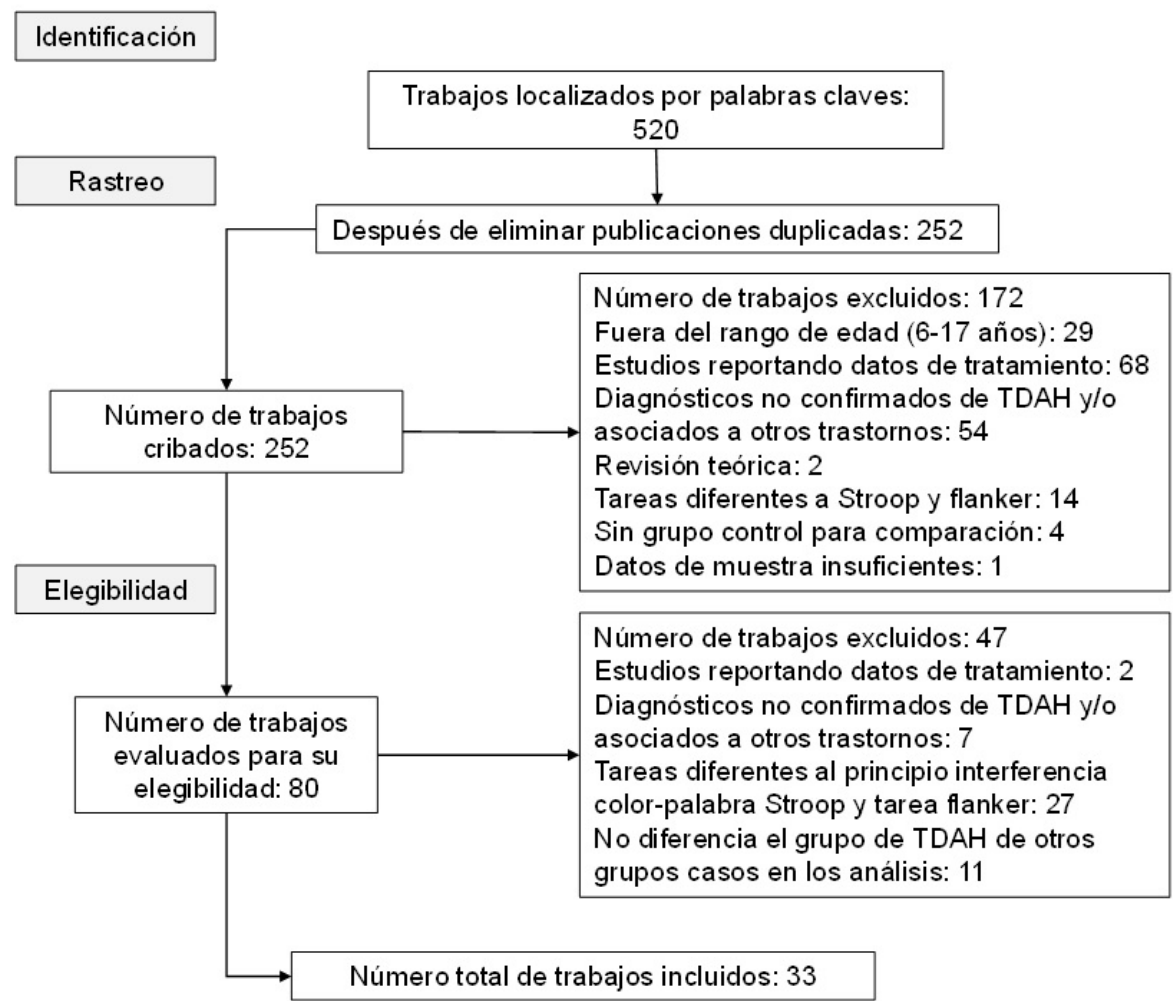

Figura 1. Diagrama de flujo del proceso de revisión.

\section{Resultados}

Los artículos fueron clasificados por el tipo de tarea utilizada (Stroop y Flanker) y los detalles de cada estudio se encuentran en las Tablas 1 y 2.

\section{Distribución geográfica de los estudios}

Geográficamente, el interés de este tema, estimado por el número de publicaciones, se encontró distribuido en países de diferentes continentes; el país que más publicaciones reportó fue China con 7 estudios, seguido de Estados Unidos con 5 y Alemania y Noruega con 3, Japón, Turquía, Italia y Francia con 2 artículos y finalmente, México, Ecuador, Brasil, Polonia, Indonesia, España y Francia con 1 publicación.

\section{Características clínicas de los participantes en los estudios}

Con relación a las características de los sujetos de estudio, de los 33 artículos, 13 reportaron comorbilidades con el TDAH, 4 de ellos con Trastorno Oposicionista Desafiante (TOD), 1 con Trastorno de Conducta (TC), 1 con Trastorno del Aprendizaje (TA), 5 con TOD+TC y 3 con TOD+TC y otros trastornos. Igualmente, 4 estudios presentaron un grupo diferenciado de TDAH con comorbilidad ( 3 con TOD y 1 con TC); 1 solo estudio tiene, además del grupo con TDAH, 1 grupo con Trastorno de Gilles de la Tourette. Con respecto al uso de medicación durante la evaluación, 7 estudios reportaron suspensión de esta; 2 estudios no solicitaron la suspensión, 9 no reportaron si los sujetos estaban en tratamiento farmacológico y 15 estudios indicaron que los sujetos no estaban en tratamiento farmacológico. 
Tabla 1. Características de los estudios sobre control de la interferencia en niños con TDAH evaluado a través de tareas tipo Stroop

\begin{tabular}{|c|c|c|c|c|c|c|c|c|}
\hline Estudio & Grupos & $\begin{array}{l}\text { Muestra } \\
\text { (M/F) }\end{array}$ & $\begin{array}{l}\text { Edad M } \\
\text { (DE) }\end{array}$ & $C I M(D E)$ & $\begin{array}{c}\text { Comorbil- } \\
\text { idad }\end{array}$ & Medicación & Tarea & $\begin{array}{l}\text { Resultados princi- } \\
\text { pales }\end{array}$ \\
\hline \multirow{2}{*}{$\begin{array}{l}\text { Bledsoe, Sem- } \\
\text { rud-Clikeman, y } \\
\text { Pliszka (2010) } \\
\text { [Estados Uni- } \\
\text { dos] }\end{array}$} & TDAH & $\begin{array}{c}40 \\
(23 / 17)\end{array}$ & $12.0(2.0)$ & -- & \multirow[b]{2}{*}{-- } & \multirow{2}{*}{$\begin{array}{l}\text { Suspendida } \\
\text { por } 24 \mathrm{~h}-48 \mathrm{~h}\end{array}$} & \multirow{2}{*}{$\begin{array}{l}\text { Tarea tipo } \\
\text { Stroop - } \\
\text { D-KEFS }\end{array}$} & \multirow{2}{*}{$\begin{array}{l}\text { Diferencias en el ti- } \\
\text { empo de ejecución en } \\
\text { las fases de denomi- } \\
\text { nación y conflicto. }\end{array}$} \\
\hline & Control & $20(11 / 9)$ & $13.0(2.0)$ & -- & & & & \\
\hline \multirow{2}{*}{$\begin{array}{l}\text { Borkowska y } \\
\text { Scholz (2010) } \\
\text { [Polonia] }\end{array}$} & TDAH & $25(23 / 2)$ & $8.10(10)$ & $\begin{array}{l}115.5 \\
(15.3)\end{array}$ & -- & \multirow{2}{*}{-- } & \multirow{2}{*}{$\begin{array}{l}\text { Tarea tipo } \\
\text { Stroop }\end{array}$} & \multirow{2}{*}{$\begin{array}{l}\text { Diferencias en el ti- } \\
\text { empo de ejecución en } \\
\text { la fase de conflicto. }\end{array}$} \\
\hline & control & $25(23 / 2)$ & $8.6(11)$ & $\begin{array}{l}113.5 \\
(14.2)\end{array}$ & -- & & & \\
\hline \multirow{3}{*}{$\begin{array}{l}\text { Christiansen y } \\
\text { Oades (2010) }\end{array}$} & TDAH & $35(32 / 3)$ & $11.2(2.4)$ & $\begin{array}{l}101.7 \\
(13.9)\end{array}$ & \multirow{3}{*}{$\begin{array}{l}\text { TOD, TC, } \\
\text { ansiedad y } \\
\text { trastornos } \\
\text { del afecto }\end{array}$} & & \multirow{3}{*}{$\begin{array}{l}\text { Tarea tipo } \\
\text { Stroop com- } \\
\text { putarizado }\end{array}$} & \multirow{3}{*}{$\begin{array}{l}\text { Diferencias en los } \\
\text { tiempos de reacción } \\
\text { en los estímulos sin } \\
\text { conflictos y en la } \\
\text { variabilidad en los } \\
\text { tiempos de reacción } \\
\text { en todas las condi- } \\
\text { ciones. }\end{array}$} \\
\hline & Hermanos & $\begin{array}{c}24 \\
(11 / 13)\end{array}$ & $11.3(3.3)$ & $\begin{array}{l}107.3 \\
(11.9)\end{array}$ & & & & \\
\hline & Control & $\begin{array}{c}37 \\
(15 / 22)\end{array}$ & 11.5 (1.9) & $108.7(2.0)$ & & & & \\
\hline \multirow{2}{*}{$\begin{array}{l}\text { Goth-Ow- } \\
\text { ens, Marti- } \\
\text { nez-Torteya, } \\
\text { Martel, y Nigg } \\
\text { (2010) }\end{array}$} & TDAH-I & $\begin{array}{c}40 \\
(27 / 13)\end{array}$ & $11.8(3.8)$ & $\begin{array}{l}104.0 \\
(13.9)\end{array}$ & \multirow{3}{*}{$\begin{array}{l}\text { TOD, TC, } \\
\text { ansiedad, } \\
\text { trastorno } \\
\text { de ánimo }\end{array}$} & \multirow{3}{*}{--} & \multirow{3}{*}{$\begin{array}{l}\text { Tarea tipo } \\
\text { Stroop } \\
\text { (Clásico y } \\
\text { D-KEFS) }\end{array}$} & \multirow{3}{*}{$\begin{array}{l}\text { Diferencias en el } \\
\text { tiempo de ejecución } \\
\text { en la fase de denom- } \\
\text { inación. }\end{array}$} \\
\hline & TDAH-C & $\begin{array}{c}66 \\
(51 / 15)\end{array}$ & $10.5(2.4)$ & $\begin{array}{l}101.3 \\
(11.7)\end{array}$ & & & & \\
\hline $\begin{array}{l}\text { [Estados Uni- } \\
\text { dos] }\end{array}$ & Control & $\begin{array}{c}276 \\
(131 / 145)\end{array}$ & $12.4(3.0)$ & $\begin{array}{l}109.4 \\
(14.1) \\
\end{array}$ & & & & \\
\hline \multirow{3}{*}{$\begin{array}{l}\text { Qian, Shuai, Cao, } \\
\text { Chan, y Wang } \\
(2010)\end{array}$} & TDAH & $\begin{array}{c}89 \\
(76 / 13)\end{array}$ & $9.0(1.9)$ & $\begin{array}{l}108.2 \\
(13.6)\end{array}$ & \multirow{3}{*}{ TOD } & \multirow{3}{*}{ No } & \multirow{3}{*}{$\begin{array}{l}\text { Tarea tipo } \\
\text { Stroop }\end{array}$} & \multirow{3}{*}{$\begin{array}{l}\text { Diferencias en tiempo } \\
\text { de ejecución en la } \\
\text { fase de conflicto. }\end{array}$} \\
\hline & TDAH+TOD & $\begin{array}{c}53 \\
(42 / 11)\end{array}$ & $9.2(1.7)$ & $\begin{array}{l}107.0 \\
(13.4)\end{array}$ & & & & \\
\hline & Control & $\begin{array}{c}116 \\
(97 / 19)\end{array}$ & $9.1(1.6)$ & $\begin{array}{l}112.8 \\
(11.5)\end{array}$ & & & & \\
\hline \multirow[t]{2}{*}{$\begin{array}{l}\text { Song y Hakoda } \\
\text { (2011) }\end{array}$} & TDAH-I & $15(11 / 4)$ & $11.0(1.4)$ & $86.7(13.4)$ & \multirow{2}{*}{ No } & \multirow{2}{*}{ No } & \multirow{2}{*}{$\begin{array}{l}\text { Tarea tipo } \\
\text { Stroop }\end{array}$} & \multirow{2}{*}{$\begin{array}{l}\text { Diferencias en la } \\
\text { precisión (aciertos/ } \\
\text { errores) en la fase de } \\
\text { conflicto. }\end{array}$} \\
\hline & Control & $15(11 / 4)$ & $\begin{array}{c}11.0 \\
(1.51)\end{array}$ & $89.0(12.7)$ & & & & \\
\hline \multirow[t]{2}{*}{$\begin{array}{l}\text { Yang et al. } \\
(2011)\end{array}$} & TDAH & $\begin{array}{c}100 \\
(90 / 10)\end{array}$ & $8.4(1.5)$ & $99.3(12.1)$ & \multirow{2}{*}{$\begin{array}{l}\text { TOD, TC, } \\
\text { TICs, prob- } \\
\text { lemas de } \\
\text { aprendizaje }\end{array}$} & \multirow[t]{2}{*}{ No } & \multirow{2}{*}{$\begin{array}{l}\text { Tarea tipo } \\
\text { Stroop (Vic- } \\
\quad \text { toria) }\end{array}$} & \multirow{2}{*}{$\begin{array}{l}\text { Diferencias en la } \\
\text { precisión (aciertos/ } \\
\text { errores) en la fase de } \\
\text { conflicto. }\end{array}$} \\
\hline & Control & $\begin{array}{c}100 \\
(89 / 11)\end{array}$ & $8.4(1.5)$ & $\begin{array}{l}106.8 \\
(11.1)\end{array}$ & & & & \\
\hline \multirow[t]{2}{*}{$\begin{array}{l}\text { Yáñez-Téllez et } \\
\text { al. (2012) }\end{array}$} & TDAH & $26(26 / 0)$ & $8.6(1.7)$ & $106.4(12)$ & \multirow{2}{*}{-- } & & $\begin{array}{l}\text { Test de pa- } \\
\text { labra-color }\end{array}$ & No se presentaron \\
\hline & Control & $25(25 / 0)$ & $9.0(1.7)$ & $\begin{array}{l}109.8 \\
(14.3)\end{array}$ & & INO & $\begin{array}{l}\text { de Stroop - } \\
\text { Clásico }\end{array}$ & grupos. \\
\hline
\end{tabular}




\begin{tabular}{|c|c|c|c|c|c|c|c|c|}
\hline Estudio & Grupos & $\begin{array}{c}\text { Muestra } \\
\text { (M/F) }\end{array}$ & $\begin{array}{l}\text { Edad M } \\
\qquad(D E)\end{array}$ & $C I M(D E)$ & $\begin{array}{l}\text { Comorbil- } \\
\text { idad }\end{array}$ & Medicación & Tarea & $\begin{array}{l}\text { Resultados princi- } \\
\text { pales }\end{array}$ \\
\hline \multirow{2}{*}{$\begin{array}{l}\text { Bledsoe, Sem- } \\
\text { rud-Clikeman, y } \\
\text { Pliszka (2013) } \\
\text { [Estados Uni- } \\
\text { dos] }\end{array}$} & TDAH & $\begin{array}{c}32 \\
(21 / 11)\end{array}$ & $11.7(1.8)$ & -- & \multirow[b]{2}{*}{--} & \multirow{2}{*}{$\begin{array}{l}\text { Suspendida } \\
\text { por } 36 \mathrm{~h}-48 \mathrm{~h}\end{array}$} & \multirow{2}{*}{$\begin{array}{l}\text { Tarea tipo } \\
\text { Stroop- } \\
\text { D-KEFS }\end{array}$} & \multirow{2}{*}{$\begin{array}{l}\text { No se presentaron } \\
\text { diferencias entre } \\
\text { grupos. }\end{array}$} \\
\hline & Control & $15(11 / 4)$ & $11.1(2.0)$ & -- & & & & \\
\hline $\begin{array}{l}\text { Borella, de Ri- } \\
\text { baupierre, Cor- } \\
\text { noldi, y Chicher- } \\
\text { io (2013) }\end{array}$ & TDAH & $24(20 / 4)$ & $9.5(1.3)$ & $100.0(6.8)$ & \multirow[t]{2}{*}{--} & \multirow[t]{2}{*}{ No } & \multirow[t]{2}{*}{$\begin{array}{l}\text { Tarea tipo } \\
\text { Stroop com- } \\
\text { putarizado }\end{array}$} & \multirow[t]{2}{*}{$\begin{array}{l}\text { No se presentaron } \\
\text { diferencias entre } \\
\text { grupos. }\end{array}$} \\
\hline [Italia] & Control & $24(16 / 8)$ & $9.2(1.4)$ & $101.8(6.2)$ & & & & \\
\hline \multirow{2}{*}{$\begin{array}{l}\text { Rubiales, Bak- } \\
\text { ker, y Urquijo } \\
(2013) \\
\text { [Argentina] }\end{array}$} & TDAH & $\begin{array}{c}30 \\
(20 / 10)\end{array}$ & $10.4(2.3)$ & $92.7(10.5)$ & \multirow{2}{*}{--} & \multirow{2}{*}{$\begin{array}{l}\text { Suspendida } \\
\text { por } 24 \mathrm{~h}\end{array}$} & \multirow{2}{*}{$\begin{array}{l}\text { Test de pa- } \\
\text { labra-color } \\
\text { de Stroop- } \\
\text { Clásico }\end{array}$} & \multirow{2}{*}{$\begin{array}{l}\text { Diferencias en la } \\
\text { precisión (aciertos/ } \\
\text { errores) en la fase de } \\
\text { conflicto. }\end{array}$} \\
\hline & Control & $\begin{array}{c}30 \\
(20 / 10)\end{array}$ & $10.9(1.5)$ & $97.6(9.6)$ & & & & \\
\hline \multirow{2}{*}{$\begin{array}{l}\text { Capelatto, Lima, } \\
\text { Ciasca, y Salga- } \\
\text { do-Azoni (2014) } \\
\text { [Brasil] }\end{array}$} & TDAH & $17(15 / 2)$ & $10.4(1.8)$ & -- & \multirow{2}{*}{--} & \multirow{2}{*}{--} & \multirow{2}{*}{$\begin{array}{l}\text { Tarea tipo } \\
\text { Stroop (Vic- } \\
\quad \text { toria) }\end{array}$} & \multirow{2}{*}{$\begin{array}{l}\text { Diferencias en la } \\
\text { precisión (aciertos/ } \\
\text { errores) y tiempos de } \\
\text { ejecución en todas } \\
\text { las fases. }\end{array}$} \\
\hline & Control & $17(10 / 7)$ & $9.4(1.0)$ & -- & & & & \\
\hline \multirow{2}{*}{$\begin{array}{l}\text { Li et al. (2014) } \\
\text { [China] }\end{array}$} & TDAH & $33(33 / 0)$ & $10.1(2.6)$ & -- & \multirow{2}{*}{-- } & \multirow{2}{*}{ No } & \multirow{2}{*}{$\begin{array}{l}\text { Tarea tipo } \\
\text { Stroop }\end{array}$} & \multirow{2}{*}{$\begin{array}{l}\text { Diferencias en la } \\
\text { precisión (aciertos/ } \\
\text { errores) y tiempos de } \\
\text { ejecución en todas } \\
\text { las fases. }\end{array}$} \\
\hline & Control & $32(32 / 0)$ & $10.9(2.6)$ & -- & & & & \\
\hline \multirow{2}{*}{$\begin{array}{l}\text { Sorensen, } \\
\text { Plessen, Adolfs- } \\
\text { dottir, y Lunder- } \\
\text { vold (2014) }\end{array}$} & TDAH & $17(--)$ & $9.5(0.9)$ & $81.4(9.7)$ & \multirow{3}{*}{$\begin{array}{l}\text { TOD, fobia } \\
\text { específica }\end{array}$} & \multirow{3}{*}{--} & \multirow{3}{*}{$\begin{array}{l}\text { Tarea tipo } \\
\text { Stroop }\end{array}$} & \multirow{3}{*}{$\begin{array}{l}\text { Diferencias en la } \\
\text { precisión (aciertos/ } \\
\text { errores) en la fase de } \\
\text { conflicto. }\end{array}$} \\
\hline & $\mathrm{TDAH}+\mathrm{COM}$ & $22(--)$ & $10.1(0.8)$ & $87.8(16.0)$ & & & & \\
\hline [Noruega] & Control & $56(--)$ & $9.7(1.0)$ & $94.8(14.2)$ & & & & \\
\hline \multirow[t]{2}{*}{$\begin{array}{l}\text { Chen et al. } \\
\text { (2015) }\end{array}$} & TDAH-C & $33(32 / 1)$ & $9.7(2.2)$ & $104.2(5.2)$ & \multirow{2}{*}{ No } & \multirow{2}{*}{ No } & \multirow{2}{*}{$\begin{array}{l}\text { Test de pa- } \\
\text { labra-color } \\
\text { de Stroop } \\
\text { (fase de con- } \\
\quad \text { flicto) }\end{array}$} & \multirow{2}{*}{$\begin{array}{l}\text { Diferencias en la } \\
\text { precisión (aciertos/ } \\
\text { errores) y tiempos de } \\
\text { ejecución. }\end{array}$} \\
\hline & Control & $35(33 / 2)$ & $10.5(1.8)$ & $107.1(6.4)$ & & & & \\
\hline $\begin{array}{l}\text { Thursina et al. } \\
\text { (2015) }\end{array}$ & TDAH & $65(58 / 7)$ & $9.4(1.7)$ & $\begin{array}{l}101.7 \\
(13.3)\end{array}$ & & No & Tarea tipo & $\begin{array}{l}\text { Diferencias en la } \\
\text { precisión (aciertos/ }\end{array}$ \\
\hline [Indonesia] & Control & $70(62 / 8)$ & $9.5(1.6)$ & $\begin{array}{l}107.7 \\
(10.6)\end{array}$ & -- & No & Stroop & $\begin{array}{l}\text { errores) y tiempos de } \\
\text { ejecución en todas } \\
\text { las fases. }\end{array}$ \\
\hline $\begin{array}{l}\text { Young et al. } \\
(2015)\end{array}$ & TDAH & $37(37 / 0)$ & $9.9(2.4)$ & -- & & & $\begin{array}{l}\text { Test de pa- } \\
\text { labra-color }\end{array}$ & $\begin{array}{l}\text { Diferencias en la } \\
\text { precisión (aciertos/ }\end{array}$ \\
\hline [China] & Control & $35(35 / 0)$ & $10.7(2.6)$ & -- & & & $\begin{array}{l}\text { (fase de con- } \\
\quad \text { flicto) }\end{array}$ & $\begin{array}{l}\text { errores) y tiempos de } \\
\text { ejecución. }\end{array}$ \\
\hline
\end{tabular}




\begin{tabular}{|c|c|c|c|c|c|c|c|c|}
\hline Estudio & Grupos & $\begin{array}{c}\text { Muestra } \\
(M / F)\end{array}$ & $\begin{array}{l}\text { Edad M } \\
\quad(D E)\end{array}$ & $C I M(D E)$ & $\begin{array}{l}\text { Comorbil- } \\
\text { idad }\end{array}$ & Medicación & Tarea & $\begin{array}{l}\text { Resultados princi- } \\
\text { pales }\end{array}$ \\
\hline \multirow{3}{*}{$\begin{array}{l}\text { Hovik, Plessen, } \\
\text { Skogli, Anders- } \\
\text { en, y Oie (2016) } \\
\text { [Noruega] }\end{array}$} & Tourette & $19(16 / 3)$ & $11.8(2.2)$ & $\begin{array}{l}101.9 \\
(15.2)\end{array}$ & \multirow{3}{*}{--} & \multirow{3}{*}{ No } & \multirow{3}{*}{$\begin{array}{l}\text { Tarea tipo } \\
\text { Stroop- } \\
\text { D-KEFS }\end{array}$} & \multirow{3}{*}{$\begin{array}{l}\text { Diferencias en la } \\
\text { precisión (aciertos/ } \\
\text { errores) en la fase } \\
\text { de conflicto y en los } \\
\text { tiempos de ejecución } \\
\text { en todas las fases. }\end{array}$} \\
\hline & TDAH & $\begin{array}{c}79 \\
(42 / 37)\end{array}$ & $11.7(2.0)$ & $95.2(14.3)$ & & & & \\
\hline & Control & $\begin{array}{c}50 \\
(32 / 18)\end{array}$ & $11.6(2.0)$ & $\begin{array}{l}103.8 \\
(12.9)\end{array}$ & & & & \\
\hline \multirow{3}{*}{$\begin{array}{l}\text { Huang et al. } \\
(2016)\end{array}$} & TDAH & $\begin{array}{c}870(713 \\
/ 157)\end{array}$ & 8.91 (1.9) & $\begin{array}{l}105.17 \\
(14.36) \\
\end{array}$ & \multirow{3}{*}{ No } & \multirow{3}{*}{ No } & \multirow{3}{*}{$\begin{array}{l}\text { Tarea tipo } \\
\text { Stroop }\end{array}$} & \multirow{3}{*}{$\begin{array}{l}\text { El grupo TDAH+TA } \\
\text { presentó un peor } \\
\text { desempeño (aciertos/ } \\
\text { errores) en compara- } \\
\text { ción con el grupo } \\
\text { TDAH y control. }\end{array}$} \\
\hline & TDAH + TA & $\begin{array}{c}1043 \\
(890 / 153)\end{array}$ & $\begin{array}{l}10.79 \\
(2.45)\end{array}$ & $\begin{array}{c}98.66 \\
(13.87)\end{array}$ & & & & \\
\hline & CONTROL & $\begin{array}{c}496 \\
(373 / 123)\end{array}$ & $9.8(1.77)$ & $\begin{array}{c}112.9 \\
(13.87)\end{array}$ & & & & \\
\hline \multirow{2}{*}{$\begin{array}{l}\text { Elosúa, Del } \\
\text { Olmo, y Con- } \\
\text { treras (2017) } \\
\text { [España] }\end{array}$} & TDAH & $26(17 / 9)$ & $9.2(0.4)$ & $95.1(1.9)$ & \multirow{2}{*}{-- } & \multirow{2}{*}{ No } & \multirow{2}{*}{$\begin{array}{l}\text { Test de pa- } \\
\text { labra-color } \\
\text { de Stroop- } \\
\text { Clásico }\end{array}$} & \multirow{2}{*}{$\begin{array}{l}\text { Diferencias en la } \\
\text { precisión (aciertos/ } \\
\text { errores) en la fase de } \\
\text { lectura. }\end{array}$} \\
\hline & Control & $\begin{array}{c}29 \\
(13 / 16)\end{array}$ & $9.1(0.4)$ & $97.1(1.7)$ & & & & \\
\hline \multirow{2}{*}{$\begin{array}{l}\text { Ramos-Galarza } \\
\text { y Perez-Salas } \\
(2017)\end{array}$} & TDAH & $\begin{array}{c}34 \\
(23 / 11)\end{array}$ & $9.8(0.5)$ & -- & \multirow{2}{*}{-- } & \multirow{2}{*}{-- } & \multirow{2}{*}{$\begin{array}{l}\text { Tarea tipo } \\
\text { Stroop (Vic- } \\
\text { toria) }\end{array}$} & \multirow{2}{*}{$\begin{array}{l}\text { Diferencias en la } \\
\text { precisión (aciertos/ } \\
\text { errores) en la fase de } \\
\text { conflicto. }\end{array}$} \\
\hline & Control & $\begin{array}{c}34 \\
(17 / 17)\end{array}$ & $9.8(0.3)$ & -- & & & & \\
\hline \multirow{3}{*}{$\begin{array}{l}\text { Uytun et al. } \\
\text { (2017) } \\
\text { [Turquía] }\end{array}$} & TDAH & 10 & -- & $\begin{array}{c}93.7 \\
(16.19)\end{array}$ & \multirow{3}{*}{$\mathrm{TC}$} & \multirow{3}{*}{ No } & \multirow{3}{*}{$\begin{array}{l}\text { Tarea tipo } \\
\text { Stroop } \\
\text { (TBAG) }\end{array}$} & \multirow{3}{*}{$\begin{array}{l}\text { Los niños con } \\
\text { TDAH+TC tuvieron } \\
\text { tiempos de ejecución } \\
\text { mayores en la fase } \\
\text { de conflicto. Los dos } \\
\text { grupos TDAH pre- } \\
\text { sentaron menor pre- } \\
\text { cisión (aciertos) en la } \\
\text { fase de conflicto. }\end{array}$} \\
\hline & TDAH+TC & 10 & -- & $\begin{array}{c}81.2 \\
(16.13)\end{array}$ & & & & \\
\hline & Control & 10 & -- & $\begin{array}{c}106 \\
(12.05)\end{array}$ & & & & \\
\hline \multirow{4}{*}{$\begin{array}{l}\text { Bahcivan, } \\
\text { Ayvasik, y Aly- } \\
\text { anak (2015) }\end{array}$} & TDAH-I & 37 & $9.9(1.7)$ & $\begin{array}{l}103.5 \\
(11.2)\end{array}$ & \multirow{4}{*}{ TOD } & \multirow{4}{*}{--} & \multirow{4}{*}{$\begin{array}{l}\text { Tarea tipo } \\
\text { Stroop } \\
\text { (TBAG) }\end{array}$} & \multirow{4}{*}{$\begin{array}{l}\text { No se encontraron } \\
\text { diferencias entre } \\
\text { grupos. }\end{array}$} \\
\hline & TDAH-C & 37 & $8.9(1.6)$ & $\begin{array}{l}103.6 \\
(11.4)\end{array}$ & & & & \\
\hline & $\mathrm{TDAH}+\mathrm{COM}$ & 37 & $9,1(1.8)$ & $\begin{array}{l}102.3 \\
(11.4)\end{array}$ & & & & \\
\hline & Control & 36 & $9,3(1,6)$ & $\begin{array}{l}108.3 \\
(11.6)\end{array}$ & & & & \\
\hline
\end{tabular}

TDAH: Trastorno por déficit de atención e hiperactividad, TDAH-C: subtipo combinado, TDAH-I: subtipo inatento; TDAH-HI: subtipo hiperactivo-impulsivo, M/F: masculino/femenino, COM: comorbilidades, TOD: Trastorno oposicionista desafiante; TC: Trastorno de conducta, PRE: potenciales evocados relacionados a eventos, Cl: Coeficiente Intelectual, D-KEFS: Delis-Kaplan Executive Function System 
Tabla 2. Características de los estudios sobre control de la interferencia en niños con TDAH evaluado a través de tareas tipo Flanker

\begin{tabular}{|c|c|c|c|c|c|c|c|c|}
\hline Estudio & Grupos & $\begin{array}{l}\text { Muestra } \\
\text { (M/F) }\end{array}$ & $\begin{array}{l}\text { Edad M } \\
\quad(D E)\end{array}$ & $C I M(D E)$ & $\begin{array}{l}\text { Comorb- } \\
\text { ilidad }\end{array}$ & $\begin{array}{l}\text { Medi- } \\
\text { cación }\end{array}$ & Tarea & Resultados principales \\
\hline \multirow[t]{2}{*}{$\begin{array}{l}\text { Yordanova et al. } \\
(2011)\end{array}$} & TDAH & $47(47 / 0)$ & $11.3(1.7)$ & $104.1(10.5)$ & \multirow{2}{*}{ TOD, TC } & \multirow{2}{*}{$\begin{array}{l}\text { Suspen- } \\
\text { dida por } \\
48 \mathrm{H}\end{array}$} & \multirow{2}{*}{$\begin{array}{l}\text { Tarea tipo } \\
\text { Flanker } \\
\text { (orientación } \\
\text { vertical) }\end{array}$} & \multirow{2}{*}{$\begin{array}{l}\text { Diferencias en la pre- } \\
\text { cisión (aciertos/errores) } \\
\text { en todas las condiciones. }\end{array}$} \\
\hline & Control & $28(28 / 0)$ & $11.1(2.0)$ & $109.5(13.5)$ & & & & \\
\hline \multirow[t]{2}{*}{$\begin{array}{l}\text { Johnstone y Gal- } \\
\text { letta (2013) }\end{array}$} & TDAH-C & $14(9 / 5)$ & $11.9(2.1)$ & $99.5(17.9)$ & \multirow{2}{*}{$\begin{array}{l}\text { Síntomas } \\
\text { TOD }\end{array}$} & \multirow{2}{*}{$\begin{array}{l}\text { Suspen- } \\
\text { dida por } \\
24 \mathrm{H}\end{array}$} & \multirow{2}{*}{$\begin{array}{l}\text { Tarea tipo } \\
\text { Flanker }\end{array}$} & \multirow{2}{*}{$\begin{array}{l}\text { Diferencias en la pre- } \\
\text { cisión (aciertos/errores) } \\
\text { en los ensayos incongru- } \\
\text { entes. }\end{array}$} \\
\hline & Control & $15(10 / 5)$ & $11.6(2.1)$ & $115.6(11.6)$ & & & & \\
\hline \multirow{2}{*}{$\begin{array}{l}\text { Rosch y Hawk } \\
\text { (2013) } \\
\text { [Estados Unidos] }\end{array}$} & TDAH & $30(24 / 6)$ & $11.2(0.8)$ & $106.1(11.9)$ & \multirow[b]{2}{*}{$\begin{array}{l}\text { Síntomas } \\
\text { de TOD } \\
\text { у TC }\end{array}$} & \multirow[b]{2}{*}{$\begin{array}{l}\text { Suspen- } \\
\text { dida por } \\
24 \mathrm{H}\end{array}$} & \multirow[b]{2}{*}{$\begin{array}{l}\text { Tarea tipo } \\
\text { Flanker, PRE }\end{array}$} & \multirow{2}{*}{$\begin{array}{l}\text { Diferencias en la pre- } \\
\text { cisión (aciertos/errores) } \\
\text { en los ensayos congru- } \\
\text { entes y en los tiempos } \\
\text { de reacción en todas las } \\
\text { condiciones. Reducción } \\
\text { de amplitud en P3 y Pe. }\end{array}$} \\
\hline & Control & $25(19 / 6)$ & $11.5(1.0)$ & $114.4(12.0)$ & & & & \\
\hline \multirow{2}{*}{$\begin{array}{l}\text { Adamo et al. } \\
(2014) \\
\text { [Italia] }\end{array}$} & TDAH & $70(62 / 8)$ & $10.0(2.0)$ & $97.0(12.0)$ & \multirow{2}{*}{$\begin{array}{l}\text { TOD, T. A., } \\
\text { ansiedad } \\
\text { general- } \\
\text { izada }\end{array}$} & \multirow[t]{2}{*}{--} & \multirow{2}{*}{$\begin{array}{l}\text { Tarea tipo } \\
\text { Flanker }\end{array}$} & \multirow{2}{*}{$\begin{array}{l}\text { Diferencias en la pre- } \\
\text { cisión (aciertos/errores) } \\
\text { en todas las condiciones }\end{array}$} \\
\hline & Control & $53(47 / 6)$ & $9.8(2.0)$ & $108.0(11.0)$ & & & & \\
\hline \multirow{2}{*}{$\begin{array}{l}\text { Mazaheri et al. } \\
\text { (2014) }\end{array}$} & TDAH-I & $17(11 / 6)$ & $14.3(1.8)$ & $115.1(15.5)$ & \multirow{3}{*}{ TOD } & \multirow{3}{*}{$\begin{array}{l}\text { Suspen- } \\
\text { dida por } \\
24 \mathrm{H}\end{array}$} & \multirow{3}{*}{$\begin{array}{l}\text { Tarea tipo } \\
\text { Flanker (con } \\
\text { señales) }\end{array}$} & \multirow{3}{*}{$\begin{array}{l}\text { Diferencias en la pre- } \\
\text { cisión (aciertos/errores) } \\
\text { en todas las condiciones. }\end{array}$} \\
\hline & TDAH-C & $17(11 / 6)$ & $14.6(1.4)$ & $110.6(12.4)$ & & & & \\
\hline [Estados Unidos] & Control & $23(14 / 9)$ & $14.9(1.7)$ & $115.0(10.7)$ & & & & \\
\hline $\begin{array}{l}\text { Fall et al. (2015) } \\
\text { [Francia] }\end{array}$ & TDAH-C & $11(8 / 3)$ & $9.8(1.7)$ & $112.5(14.4)$ & -- & No & $\begin{array}{l}\text { Tarea tipo } \\
\text { Flanker } \\
\text { (peces) }\end{array}$ & $\begin{array}{l}\text { Diferencias en la pre- } \\
\text { cisión (aciertos/errores) } \\
\text { en los ensayos incongru- } \\
\text { entes y en los tiempos } \\
\text { de reacción en todas las } \\
\text { condiciones. }\end{array}$ \\
\hline $\begin{array}{l}\text { Bluschke, Ch- } \\
\text { mielewski, Roess- }\end{array}$ & TDAH-C & $18(16 / 2)$ & $11.1(1.9)$ & $102(10)$ & & 17 & Tarea tipo & Diferencias en la pre- \\
\hline [Alemania] & Control & $17(7 / 10)$ & $13.5(1.9)$ & $118(13)$ & & ו & Fanker & en todas las condiciones. \\
\hline $\begin{array}{l}\text { Plessen et al. } \\
\text { (2016) }\end{array}$ & TDAH & $25(17 / 8)$ & $\begin{array}{l}10.75 \\
(1.09) \\
\end{array}$ & $92.4(5.6)$ & TC TC & $\mathrm{No}$ & Tarea tipo & $\begin{array}{l}\text { Diferencias en la pre- } \\
\text { cisión (aciertos/errores) }\end{array}$ \\
\hline [Noruega] & Control & $\begin{array}{c}29 \\
(15 / 14)\end{array}$ & $\begin{array}{l}10.15 \\
(1.04)\end{array}$ & $106.4(11.4)$ & שו הטתו & (10 & Flanker & $\begin{array}{l}\text { y tiempos de reacción en } \\
\text { todas las condiciones. }\end{array}$ \\
\hline $\begin{array}{l}\text { Suzuki et al. } \\
(2017)\end{array}$ & TDAH & $12(11 / 1)$ & $9.9(1.0)$ & -- & & & Tarea tipo & $\begin{array}{l}\text { Diferencias en la pre- } \\
\text { cisión (aciertos/errores) }\end{array}$ \\
\hline [Japón] & Control & $14(10 / 4)$ & $9.5(1.0)$ & -- & & & Flanker & $\begin{array}{l}\text { en los ensayos incongru- } \\
\text { entes. }\end{array}$ \\
\hline
\end{tabular}

TDAH: Trastorno por déficit de atención e hiperactividad, TDAH-C: subtipo combinado, TDAH-I: subtipo inatento; TDAH-HI: subtipo hiperactivo-impulsivo, M/F: masculino/femenino, COM: comorbilidades, PRE: potenciales evocados relacionados a eventos, TOD: Trastorno oposicionista desafiante; TC: trastorno de conducta; T.A.: trastorno de aprendizaje; CI: Coeficiente Intelectual 
Pág 113

Uno de los aspectos más evaluados en el control de la interferencia fue la precisión en la ejecución de la tarea, que incluye los aciertos y errores (omisión y comisión) cometidos. De los 33 estudios seleccionados, 21 de ellos reportaron que los niños con TDAH presentaron un desempeño menor en comparación a los niños sin TDAH.

\section{Tipo de versiones de las tareas utilizadas}

De los estudios seleccionados, 23 de ellos utilizaron una tarea tipo Stroop y 10 una tarea tipo Flanker. Entre las modificaciones más usadas de la tarea tipo Stroop se encontró una que incluye una cuarta fase (color-palabra congruente o palabras neutras, seis estudios) y entre esas se destacó la subprueba perteneciente al Delis-Kaplan Executive Function System (D-KEFS) (3 estudios); otra de las versiones más comunes fue la versión Victoria utilizada por tres estudios, la clásica de 45 segundos (3 estudios) y la clásica con modificación del tiempo límite de 45 segundos a tiempo de ejecución (3 estudios); 2 estudios usaron solo la fase de conflicto de la versión clásica, 2 usaron la versión TBAG que consta de cinco fases, 2 utilizaron una versión con dos fases (congruente e incongruente; uno computarizado) y 1 computarizado con tres condiciones (congruente, incongruente, neutro); de los 23 estudios solamente 5 se realizaron en sistemas computarizados, y los 18 restantes en hojas de papel o láminas. Es de resaltar que 1 estudio (Goth-Owens et al., 2010) utilizó tanto la versión clásica como la subprueba del D-KEFS. Con relación a las tareas tipo Flanker, 8 de 10 estudios utilizaron una versión tradicional de la tarea al utilizar flechas ubicadas horizontalmente como estímulos; de los otros 2 estudios, 1 utilizó peces como estímulo y otro ubicó los estímulos distractores arriba y abajo del estímulo diana.

\section{Precisión en las tareas}

Uno de los aspectos más evaluados en el control de la interferencia fue la precisión en la ejecución de la tarea, que incluye los aciertos y errores (omisión y comisión) cometidos. De los 33 estudios seleccionados, 21 de ellos reportaron que los niños con TDAH presentaron un desempeño menor en comparación a los niños sin TDAH (Adamo et al., 2014; Bluschke et al., 2016; Capelatto et al., 2014; Chen et al., 2015; Elosúa et al., 2017; Fall et al., 2015; Hovik et al., 2016; Johnstone \& Galletta, 2013; Li et al., 2014; Plessen et al., 2016; Ramos-Galarza \& Perez-Salas, 2017; Rosch \& Hawk, 2013; Rubiales et al., 2013; Song \& Hakoda, 2011; Sorensen et al., 2014; Suzuki et al., 2017; Thursina et al., 2015; Uytun et al., 2017; Yang et al., 2011; Yordanova et al., 2011; Young et al., 2015), y aunque estos errores son comunes en todas las condiciones de las tareas (con o sin conflicto, ensayos congruentes/incongruentes), son más frecuentes en los ensayos en los que se presentan conflictos o incongruencia de estímulos.

\section{Tiempo de ejecución/reacción}

En cuanto a los resultados en los tiempos de ejecución/reacción, 18 estudios reportaron diferencias estadísticamente significativas en la mayoría de los estudios a favor de los niños de los grupos controles (Bledsoe et al., 2010; Borkowska \& Scholz, 2010; Capelatto et al., 2014; Chen et al., 2015; Christiansen \& Oades, 2010; Fall et al., 2015; Fassbender et al., 2015; Goth-Owens et al., 2010; Hovik et al., 2016; Huang et al., 2016; Li et al., 2014; Mazaheri et al., 2014; Plessen et al., 2016; Qian et al., 2010; Rosch \& Hawk, 2013; Thursina et al., 2015; Uytun et al., 2017; Young et al., 2015), y lo mismo que en la precisión de las tareas, las diferencias en los tiempos de reacción/ejecución y su variabilidad son más significativas en las fases de conflicto, interferencia e incongruencia. 3 estudios utilizaron la versión clásica de la tarea Stroop (Elosúa et al., 2017; Rubiales et al., 2013; Yáñez-Téllez et al., 2012), por lo que el tiempo de ejecución/ reacción no se contempló como variable de estudio. Igualmente, 3 estudios no reportaron datos de tiempo de ejecución/reacción, aunque no utilizaron la versión clásica de la tarea Stroop (Ramos-Galarza \& Perez-Salas, 2017; Song \& Hakoda, 2011; Yang et al., 2011). 
Pág 114

Las investigaciones analizadas reportan que los niños con TDAH presentan dificultades en el control de la interferencia que se manifiestan en mayor porcentaje de errores y tiempos de reacción más amplios con mucha variabilidad. Sin embargo, algunos estudios señalan que, a pesar de presentar estas deficiencias, las diferencias no son lo suficientemente significativas como para identificar los niños que presentan TDAH de aquellos que no lo tienen (Bahcivan et al., 2015; Bledsoe et al., 2013).
De forma general, 4 estudios no encontraron diferencias significativas entre grupos en el desempeño (precisión y el tiempo de ejecución/reacción), aunque reportaron una tendencia de los niños con TDAH a presentar un desempeño más bajo que los del grupo control (Bahcivan et al., 2015; Bledsoe et al., 2013; Borella et al., 2013; Yáñez-Téllez et al., 2012).

\section{Técnicas de neuroimagen y neurofisiológicas}

Entre los estudios que además utilizaron técnicas de neuroimagen y neurofisiológicas, Fassbender et al. (2015) encontraron que en los ensayos incongruentes en la tarea tipo Flanker se presentó una activación en la corteza frontal bilateral, el área motora suplementaria y los ganglios basales en los dos grupos, sin embargo, en la preparación de respuestas ante estímulos incongruentes los niños del grupo de control, a diferencia de los niños con TDAH, presentaron una activación en el lóbulo parietal superior de forma bilateral. Con respecto a la medición neurofisiológica realizada por medio de potenciales evocados relacionados a eventos (PREs), algunos autores reportaron una reducción en la amplitud de la onda P3 relacionada con el procesamiento del estímulo de la tarea tipo Flanker, así como reducciones en las amplitudes de las ondas ERN y Pe, relacionadas con el procesamiento del error en los niños con TDAH en comparación a los niños del grupo de control (Rosch \& Hawk, 2013).

\section{Discusión}

Esta revisión tuvo como objetivo identificar la literatura científica disponible sobre el desempeño en tareas que evalúan el control de la interferencia en niños con TDAH. En este sentido, controlar adecuadamente la interferencia de estímulos irrelevantes centrándose en un atributo de un estímulo ignorando los distractores que pueden activar una respuesta competitiva es una función central de las FE (Posner \& DiGirolamo, 1998), la cual parece estar comprometida en el TDAH. Las investigaciones analizadas reportan que los niños con TDAH presentan dificultades en el control de la interferencia que se manifiestan en mayor porcentaje de errores y tiempos de reacción más amplios con mucha variabilidad. Sin embargo, algunos estudios señalan que, a pesar de presentar estas deficiencias, las diferencias no son lo suficientemente significativas como para identificar los niños que presentan TDAH de aquellos que no lo tienen (Bahcivan et al., 2015; Bledsoe et al., 2013).

Otras revisiones y metaanálisis sobre el tema han reportado que las personas con TDAH presentan un pobre control de la interferencia. Lansbergen et al. (2007) realizaron un meta-análisis seleccionando los estudios que utilizaron todas las versiones del test de Stroop, excluyendo aquellos que reportaron solo el índice de interferencia del método de calificación de Golden (Golden, 1994), y plantean que la ausencia del control de la interferencia manifestada en otros meta-análisis (Homack \& Riccio, 2004) se debe a la inclusión del índice de interferencia de Golden como único dato de análisis, a la falta de control de los tiempos de reacción ítem por ítem y del tiempo de ejecución en la tarea. En esta misma línea, Mullane et al. (2009), para confirmar lo planteado por Lansbergen et al. (2007), adelantaron una revisión seleccionando las investigaciones que utilizaron tareas tipo Flanker y tipo Simon, y encontraron que los niños con TDAH son generalmente más lentos en responder, cometen mayores errores y son menos eficientes en su desempeño en la mayoría de las condiciones de las tareas.

Algunos autores plantean que las diferencias encontradas en los resultados de las diferentes pruebas se deben a inconsistencias metodológicas, entre ellas, la utilización de muestras pequeñas con poder estadístico modesto, variaciones en la dificultad 
Pág 115

Este estudio identifica y analiza investigaciones que han evaluado el control de la interferencia en niños con TDAH utilizando tareas tipo Stroop y Flanker; de lo que se deduce que el control de la interferencia se encuentra alterado en niños con TDAH, con una marcada tendencia a presentar menores aciertos, mayores errores y tiempos de reacción/ejecución mayor que los niños sin TDAH. de las tareas y duración, amplios rangos de edades que contribuyen a variabilidades intragrupos y la dificultad para controlar o excluir trastornos comórbidos (Cao et al., 2013; Rosch \& Hawk, 2013; Thursina et al., 2015). De forma específica, se ha sugerido que las diferencias encontradas en los estudios que han usado tareas tipo Stroop se relacionan más con la presencia de problemas de lectura (Thursina et al., 2015), que con dificultades en el control de la interferencia o atencionales; sin embargo, Huang et al. (2016) compararon niños con TDAH, TDAH y problemas de lectura (PL) y niños del grupo de control y observaron que los grupos casos presentaron déficits más significativos que los niños en el grupo control y que los niños con TDAH y PL presentaron mayor déficit en el control de la interferencia que los niños con solo TDAH, y los niños con solo TDAH se diferenciaron claramente de los niños del grupo control, lo que ayuda a soportar la deficiencia en el control de la interferencia en el TDAH.

\section{Limitaciones}

Fueron limitaciones del presente estudio la heterogeneidad de los estudios analizados en términos de características de las muestras (relación sexos y comorbilidades, especialmente) y de las versiones de los instrumentos utilizados, en particular de la tarea tipo Stroop. Además, algunos de los estudios incluidos en la revisión no reportaron datos que, aunque no tuvieran una significancia estadística, podrían haber brindado información más completa sobre el desempeño en general de los participantes en los mismos.

\section{Conclusión}

Este estudio identifica y analiza investigaciones que han evaluado el control de la interferencia en niños con TDAH utilizando tareas tipo Stroop y Flanker; de lo que se deduce que el control de la interferencia se encuentra alterado en niños con TDAH, con una marcada tendencia a presentar menores aciertos, mayores errores y tiempos de reacción/ejecución mayor que los niños sin TDAH. Los estudios sugieren además alteraciones en los patrones de activación neuronal en niños con TDAH en la corteza prefrontal relacionada directamente con las FE y control inhibitorio; y variaciones en las amplitudes de los PREs relacionados con el procesamiento del estímulo, y monitoreo y resolución del conflicto. Estos resultados soportan la presencia de alteraciones en el control de la interferencia que podría repercutir en el comportamiento y desempeño académico de los niños con TDAH.

Se sugiere que en futuras investigaciones se combinen diferentes instrumentos en la evaluación del control de la interferencia en niños con TDAH con el fin de dar mayor robustez a los resultados. De igual forma, se recomienda incluir la variable de tiempo de ejecución/reacción en la evaluación del control de la interferencia en niños con TDAH, por ser considerada un endofenotipo característico del Trastorno, y un elemento primordial en las actividades que rodean a los niños con TDAH, en las que se les exige, además de un buen desempeño, realizar las tareas en el menor tiempo posible. Lo anterior permitiría fortalecer las acciones orientadas a la habilitación psicológica y neuropsicológica relacionadas a las exigencias académicas en los niños con TDAH, especialmente en lo referente al control de la interferencia. 


\section{Referencias}

Adamo, N., Di Martino, A., Esu, L., Petkova, E., Johnson, K., Kelly, S., . . Zuddas, A. (2014). Increased Response-Time Variability Across Different Cognitive Tasks in Children With ADHD. Journal of Attention Disorders, 18(5), 434-446. doi: https:// doi.org/10.1177\%2F1087054712439419

Alderson, R. M., Rapport, M. D., \& Kofler, M. J. (2007). Attention-deficit/hyperactivity disorder and behavioral inhibition: a meta-analytic review of the stop-signal paradigm. Journal of Abnormal Child Psychology, 35(5), 745-758. doi: https://doi. org/10.1007/s10802-007-9131-6

American Psychiatric Association. (2013). Diagnostic and statistical manual of mental disorders (DSM-5 ${ }^{\circledR}$ (5 ed.). Washington, D.C.: American Psychiatric Association.

Bahcivan, R., Ayvasik, H. B., \& Alyanak, B. (2015). Executive Functioning in Subtypes of Attention Deficit Hyperactivity Disorder. Noro Psikiyatri Arsivi, 52(4), 386-392. doi: https://dx.doi.org/10.5152\%2Fnpa.2015.8712

Bará-Jiménez, S., Vicuña, P., Pineda, D., \& Henao, G. C. (2003). Perfiles neuropsicológicos y conductuales de niños con trastorno por déficit de atención/hiperactividad de Cali, Colombia. Revista de neurología, 37(07), 0608-0615. doi: https://doi.org/10.33588/ rn.3707.2003189

Barkley, R. A. (1997). Behavioral inhibition, sustained attention, and executive functions: constructing a unifying theory of ADHD. Psychological Bulletin, 121(1), 65-94. doi: https://doi.org/10.1037/0033-2909.121.1.65

Bianchini, R., Postorino, V., Grasso, R., Santoro, B., Migliore, S., Burlò, C., . . Mazzone, L. (2013). Prevalence of ADHD in a sample of Italian students: A population-based study. Research in developmental disabilities, 34(9), 2543-2550. doi: https://doi. org/10.1016/j.ridd.2013.05.027

Bledsoe, J. C., Semrud-Clikeman, M., \& Pliszka, S. R. (2010). Response inhibition and academic abilities in typically developing children with attention-deficit-hyperactivity disorder-combined subtype. Archives of Clinical Neuropsychology, 25(7), 671-679. doi: https://doi.org/10.1093/arclin/acq048

Bledsoe, J. C., Semrud-Clikeman, M., \& Pliszka, S. R. (2013). Anterior cingulate cortex and symptom severity in attention-deficit/hyperactivity disorder. Journal of $A b-$ normal Psychology, 122(2), 558-565. doi: https://doi.org/10.1037/a0032390

Bluschke, A., Chmielewski, W. X., Roessner, V., \& Beste, C. (2016). Intact Context-Dependent Modulation of Conflict Monitoring in Childhood ADHD. Journal of Attention Disorders. doi: https://doi.org/10.1177/1087054716643388

Borella, E., de Ribaupierre, A., Cornoldi, C., \& Chicherio, C. (2013). Beyond interference control impairment in ADHD: evidence from increased intraindividual variability in the color-stroop test. Child Neuropsychology, 19(5), 495-515. doi: https://doi.or $\mathrm{g} / 10.1080 / 09297049.2012 .696603$

Borkowska, A. R., \& Scholz, B. (2010). Selected executive functions in children with ADHD in early school age. Psychiatria i Psychologia Kliniczna, 10(3), 141-154. Recuperado de: https://www.scopus.com/inward/record. uri?eid $=2-$ s2.0 $-79551613541 \&$ partner $\mid D=40 \& \mathrm{md} 5=0818 \mathrm{bd} 962 \mathrm{~d} 69 \mathrm{adf} 95 \mathrm{bbf}-$ b168a541d592

Cao, J., Wang, S., Ren, Y., Zhang, Y., Cai, J., Tu, W., . . Xia, Y. (2013). Interference control in 6-11 year-old children with and without ADHD: behavioral and ERP study. International Journal of Developmental Neuroscience, 31(5), 342-349. doi: https:// doi.org/10.1016/j.ijdevneu.2013.04.005

Capelatto, I. V., Lima, R. F. d., Ciasca, S. M., \& Salgado-Azoni, C. A. (2014). Cognitive Functions, Self-Esteem and Self-Concept of Children with Attention Deficit and Hyperactivity Disorder. Psicologia: Reflexão e Crítica, 27(2), 331-340. doi: https:// doi.org/10.1590/1678-7153.201427214 
Chen, L., Huang, X., Lei, D., He, N., Hu, X., Chen, Y., ... Gong, Q. Y. (2015). Microstructural abnormalities of the brain white matter in attention-deficit/hyperactivity disorder. Journal of Psychiatry \& Neuroscience, 40(4), 280-287. doi: https://doi. org/10.1503/jpn.140199

Christiansen, H., \& Oades, R. D. (2010). Negative priming within a stroop task in children and adolescents with attention-deficit hyperactivity disorder, their siblings, and independent controls. Journal of Attention Disorders, 13(5), 497-504. doi: https://doi.org/10.1177/1087054708325974

Cornejo-Escatell, E., Fajardo-Fregoso, B. F., López-Velázquez, V. M., Soto-Vargas, J., \& Ceja-Moreno, H. (2015). Prevalencia de déficit de atención e hiperactividad en escolares de la zona noreste de Jalisco, México. Revista Médica MD, 6(3), 189195. Recuperado de: https://www.medigraphic.com/pdfs/revmed/md-2015/ md153d.pdf

Cornejo, J. W., Osío, O., Sánchez, Y., Carrizosa, J., Sánchez, G., Grisales, H., . . Holguín, J. (2005). Prevalencia del trastorno por déficit de atención-hiperactividad en niños y adolescentes colombianos. Revista de neurología, 40(12), 716-722. doi: https://doi.org/10.33588/rn.4012.2004569

Crosbie, J., Perusse, D., Barr, C. L., \& Schachar, R. J. (2008). Validating psychiatric endophenotypes: inhibitory control and attention deficit hyperactivity disorder. Neuroscience \& Biobehavioral Reviews, 32(1), 40-55. doi: https://doi.org/10.1016/j. neubiorev.2007.05.002

Cruz, L. E., Ramos, A., Gutiérrez, M. F., Gutiérrez, D. E., Márquez, A. V., Ramírez, D. C., ... Sepúlveda, K. G. (2010). Prevalencia del trastorno por déficit de atención e hiperactividad en escolares de tres poblaciones del estado de Jalisco. Revista Mexicana de Neurociencia, 11(1), 15-19. Recuperado de: http://repositorio.cualtos.udg.mx:8080/jspui/bitstream/123456789/271/1/Prevalencia\%20del\%20 trastorno\%20por\%20d\%C3\%A9ficit\%20de\%20atenci\%C3\%B3n.pdf

Elosúa, M. R., Del Olmo, S., \& Contreras, M. J. (2017). Differences in Executive Functioning in Children with Attention Deficit and Hyperactivity Disorder (ADHD). Frontiers in Psychology, 8, 976. doi: https://doi.org/10.3389/fpsyg.2017.00976

Fall, S., Querne, L., Le Moing, A. G., \& Berquin, P. (2015). Individual differences in subcortical microstructure organization reflect reaction time performances during a Flanker task: a diffusion tensor imaging study in children with and without ADHD. Psychiatry Research, 233(1), 50-56. doi: https://doi.org/10.1016/j.pscychresns.2015.05.001

Fassbender, C., Krafft, C. E., \& Schweitzer, J. B. (2015). Differentiating SCT and inattentive symptoms in ADHD using fMRI measures of cognitive control. Neurolmage: Clinical, 8, 390-397. doi: https://doi.org/10.1016/j.nicl.2015.05.007

Friedman, N. P., \& Miyake, A. (2004). The relations among inhibition and interference control functions: a latent-variable analysis. Journal of Experimental Psychology: General, 133(1), 101-135. doi: https://doi.org/10.1037/0096-3445.133.1.101

Golden, C. J. (1994). STROOP: Test de colores y palabras: Manual. España: TEA ediciones.

Gómez-Betancur, L. Á., Pineda, D., \& Aguirre-Acevedo, D. C. (2005). Conciencia fonológica en niños con trastorno de la atención sin dificultades en el aprendizaje. Revista de neurología, 40(10), 0581-0586. doi: https://doi.org/10.33588/ rn.4010.2004109

Goos, L. M., Crosbie, J., Payne, S., \& Schachar, R. (2009). Validation and extension of the endophenotype model in ADHD patterns of inheritance in a family study of inhibitory control. American Journal of Psychiatry, 166(6), 711-717. doi: https:// doi.org/10.1176/appi.ajp.2009.08040621 
Goth-Owens, T. L., Martinez-Torteya, C., Martel, M. M., \& Nigg, J. T. (2010). Processing speed weakness in children and adolescents with non-hyperactive but inattentive ADHD (ADD). Child Neuropsychology, 16(6), 577-591. doi: https://doi.org/10.1 $\underline{080 / 09297049.2010 .485126}$

Homack, S., \& Riccio, C. A. (2004). A meta-analysis of the sensitivity and specificity of the Stroop Color and Word Test with children. Archives of Clinical Neuropsychology, 19(6), 725-743. doi: https://doi.org/10.1016/j.acn.2003.09.003

Hovik, K. T., Plessen, K. J., Skogli, E. W., Andersen, P. N., \& Oie, M. (2016). Dissociable Response Inhibition in Children With Tourette's Syndrome Compared With Children With ADHD. Journal of Attention Disorders, 20(10), 825-835. doi: https://doi. org/10.1177/1087054713512371

Huang, F., Sun, L., Qian, Y., Liu, L., Ma, Q. G., Yang, L., . . Wang, Y. F. (2016). Cognitive Function of Children and Adolescents with Attention Deficit Hyperactivity Disorder and Learning Difficulties: A Developmental Perspective. Chinese Medical Journal, 129(16), 1922-1928. doi: https://doi.org/10.4103/0366-6999.187861

Jimenez-Figueroa, G., Ardila-Duarte, C., Pineda, D. A., Acosta-Lopez, J. E., Cervantes-Henriquez, M. L., Pineda-Alhucema, W., ... Puentes-Rozo, P. J. (2017). Prepotent response inhibition and reaction times in children with attention deficit/hyperactivity disorder from a Caribbean community. ADHD Attention Deficit and Hyperactivity Disorders, 9(4), 199-211. doi: https://doi.org/10.1007/s12402-017-0223-z

Johnstone, S. J., \& Galletta, D. (2013). Event-rate effects in the Flanker task: ERPs and task performance in children with and without AD/HD. International Journal of Psychophysiology, 87(3), 340-348. doi: https://doi.org/10.1016/i.ijpsycho.2012.07.170

Lansbergen, M. M., Kenemans, J. L., \& van Engeland, H. (2007). Stroop interference and attention-deficit/hyperactivity disorder: a review and meta-analysis. Neuropsychology, 21(2), 251-262. doi: https://doi.org/10.1037/0894-4105.21.2.251

Li, F., He, N., Li, Y., Chen, L., Huang, X., Lui, S., ... Gong, Q. (2014). Intrinsic brain abnormalities in attention deficit hyperactivity disorder: a resting-state functional MR imaging study. Radiology, 272(2), 514-523. doi: https://doi.org/10.1148/radiol.14131622

Lijffijt, M., Kenemans, J. L., Verbaten, M. N., \& van Engeland, H. (2005). A meta-analytic review of stopping performance in attention-deficit/hyperactivity disorder: deficient inhibitory motor control? Journal of Abnormal Psychology, 114(2), 216-222. doi: https://doi.org/10.1037/0021-843X.114.2.216

Llanos, L., García, D., González, H., \& Puentes-Rozo, P. J. (2019). Trastorno por déficit de atención e hiperactivididad (TDAH) en niños escolarizados de 6 a 17 años. Revista pediatría de atención primaria, 21(83), e101-e108. Recuperado de: https:// pap.es/articulo/12844/trastorno-por-deficit-de-atencion-e-hiperactividadtdah-en-ninos-escolarizados-de-6-a-17-anos

Ma, I., Mies, G. W., Lambregts-Rommelse, N. N. J., Buitelaar, J. K., Cillessen, A. H. N., \& Scheres, A. (2018). Does an attention bias to appetitive and aversive words modulate interference control in youth with ADHD? Child Neuropsychology, 24(4), 541-557. doi: https://doi.org/10.1080/09297049.2017.1296940

Mazaheri, A., Fassbender, C., Coffey-Corina, S., Hartanto, T. A., Schweitzer, J. B., \& Mangun, G. R. (2014). Differential oscillatory electroencephalogram between attention-deficit/hyperactivity disorder subtypes and typically developing adolescents. Biological Psychiatry, 76(5), 422-429. doi: https://doi.org/10.1016/j.biopsych.2013.08.023

Moher, D., Liberati, A., Tetzlaff, J., Altman, D. G., \& Prisma Group. (2009). Preferred Reporting Items for Systematic Reviews and Meta-Analyses: The PRISMA Statement. PLOS Medicine, 6(7), e1000097. doi: https://doi.org/10.1371/journal.pmed.1000097 
Mullane, J. C., Corkum, P. V., Klein, R. M., \& McLaughlin, E. (2009). Interference control in children with and without ADHD: a systematic review of Flanker and Simon task performance. Child Neuropsychology, 15(4), 321-342. doi: https:// doi.org/10.1080/09297040802348028

Nigg, J. T. (2000). On inhibition/disinhibition in developmental psychopathology: views from cognitive and personality psychology and a working inhibition taxonomy. Psychological Bulletin, 126(2), 220-246. doi: https://doi.org/10.1037//00332909.126.2.220

Oosterlaan, J., Logan, G. D., \& Sergeant, J. A. (1998). Response inhibition in AD/HD, CD, comorbid AD/HD + CD, anxious, and control children: a meta-analysis of studies with the stop task. Journal of Child Psychology and Psychiatry, 39(3), 411-425. doi: https://doi.org/10.1111/1469-7610.00336

Pennington, B. F., \& Ozonoff, S. (1996). Executive Functions and Developmental Psychopathology. Journal of Child Psychology and Psychiatry, 37(1), 51-87. doi: https://doi.org/10.1111/j.1469-7610.1996.tb01380.x

Pineda, D. A., Ardila, A., \& Rosselli, M. (1999). Neuropsychological and behavioral assessment of ADHD in seven- to twelve-year-old children: a discriminant analysis. Journal of Learning Disabilities, 32(2), 159-173. doi: https://doi. org/10.1177/002221949903200206

Pineda, D. A., Ardila, A., Rosselli, M., Cadavid, C., Mancheno, S., \& Mejia, S. E. (1998). Executive Dysfunctions in Children with Attention Deficit Hyperactivity Disorder. International Journal of Neuroscience, 96(3-4), 177-196. doi: https://doi. org/10.3109/00207459808986466

Pineda, D. A., Lopera, F., Henao, G. C., Palacio, J. D., Castellanos, F. X., \& Fundema, G. D. I. (2001). Confirmación de la alta prevalencia del trastorno por déficit de atención en una comunidad colombiana. Revista de neurología, 32(3), 217-222. Recuperado de: https://www.neurologia.com/articulo/2000499

Plessen, K. J., Allen, E. A., Eichele, H., van Wageningen, H., Hovik, M. F., Sorensen, L., ... Eichele, T. (2016). Reduced error signalling in medication-naive children with ADHD: associations with behavioural variability and post-error adaptations. Journal of Psychiatry \& Neuroscience, 41(2), 77-87. doi: https://doi.org/10.1503/ ipn.140353

Posner, M. I., \& DiGirolamo, G. J. (1998). Conflict, target detection and cognitive control. In R. Parasuraman (Ed.), The Attentive Brain (pp. 401-423). Cambridge, MA: The MIT Press.

Puentes-Rozo, P., Acosta-López, J., Cervantes-Henríquez, M. L., Jiménez-Figueroa, G., Sánchez-Rojas, M., Pineda-Alhucema, W., \& Téllez-Bustillo, S. (2015). Neuropsicología de las Funciones Ejecutivas(2 ed.). Recuperado de: http://bonga.unisimon. edu.co/handle/20.500.12442/1209

Qian, Y., Shuai, L., Cao, Q., Chan, R. C., \& Wang, Y. (2010). Do executive function deficits differentiate between children with attention deficit hyperactivity disorder (ADHD) and ADHD comorbid with oppositional defiant disorder? A cross-cultural study using performance-based tests and the behavior rating inventory of executive function. The Clinical Neuropsychologist, 24(5), 793-810. doi: https://doi.org/10.1080/13854041003749342

Ramos-Galarza, C., \& Perez-Salas, C. (2017). Control inhibitorio y monitorización en población infantil con TDAH. Avances en Psicologia Latinoamericana, 35(1), $117-$ 130. doi: https://doi.org/10.12804/revistas.urosario.edu.co/apl/a.4195

Rosch, K. S., \& Hawk, L. W., Jr. (2013). The effects of performance-based rewards on neurophysiological correlates of stimulus, error, and feedback processing in children with ADHD. Psychophysiology, 50(11), 1157-1173. doi: https://doi. org/10.1111/psyp.12127 
Rubiales, J., Bakker, L., \& Urquijo, S. (2013). Estudio comparativo del control inhibitorio y la flexibilidad cognitiva en niños con Trastorno por déficit de atención con hiperactividad. Cuadernos de Neuropsicología, 7(1), 50-69. Recuperado de: https:// dialnet.unirioja.es/servlet/articulo?codigo $=4395899$

Ryan, R., Synnot, A., Prictor, M., \& Hill, S. (2016). Cochrane consumers and communication group data extraction template for included studies. La Trobe University, Melbourne. doi: https://doi.org/10.26181/5b57cfd711743

Schachar, R. J., Crosbie, J., Barr, C. L., Ornstein, T. J., Kennedy, J., Malone, M., . . . Pathare, T. (2005). Inhibition of motor responses in siblings concordant and discordant for attention deficit hyperactivity disorder. American Journal of Psychiatry, 162(6), 1076-1082. doi: https://doi.org/10.1176/appi.ajp.162.6.1076

Song, Y., \& Hakoda, Y. (2011). An asymmetric stroop/reverse-stroop interference phenomenon in ADHD. Journal of Attention Disorders, 15(6), 499-505. doi: https:// doi.org/10.1177/1087054710367607

Sorensen, L., Plessen, K. J., Adolfsdottir, S., \& Lundervold, A. J. (2014). The specificity of the Stroop interference score of errors to ADHD in boys. Child Neuropsychology, 20(6), 677-691. doi: https://doi.org/10.1080/09297049.2013.855716

Suzuki, K., Okumura, Y., Kita, Y., Oi, Y., Yamashita, Y., Goto, T., \& Inagaki, M. (2017). Excessive hemodynamic activity in the superior frontal cortex during the Flanker task in children with attention deficit hyperactivity disorder. Neuroreport, 28(13), 828-832. doi: https://doi.org/10.1097/WNR.0000000000000834

Thursina, C., Ar Rochmah, M., Nurputra, D. K., Harahap, I. S., Harahap, N. I., Sa'Adah, N., ... Kitayama, S. (2015). Attention Deficit/Hyperactivity Disorder (ADHD): age related change of completion time and error rates of Stroop test. Kobe Journal of Medical Sciences, 61(1), E19-26. Recuperado de: https://www.ncbi.nlm.nih.gov/ pubmed/25868610

Trujillo-Orrego, N., Ibanez, A., \& Pineda, D. (2012). Validez del diagnóstico de trastorno por déficit de atención/hiperactividad: de lo fenomenológico a lo neurobiológico (II). Revista de neurología, 54(6), 367-379. Recuperado de: https://www.neurologia.com/articulo/2011144

Uytun, M. C., Karakaya, E., Oztop, D. B., Gengec, S., Gumus, K., Ozmen, S., . . Ozsoy, S. D. (2017). Default mode network activity and neuropsychological profile in male children and adolescents with attention deficit hyperactivity disorder and conduct disorder. Brain Imaging and Behavior, 11(6), 1561-1570. doi: https://doi. org/10.1007/s11682-016-9614-6

Van Mourik, R., Oosterlaan, J., \& Sergeant, J. A. (2005). The Stroop revisited: a meta-analysis of interference control in AD/HD. Journal of Child Psychology and Psychiatry, 46(2), 150-165. doi: https://doi.org/10.1111/j.1469-7610.2004.00345.x

van Mourik, R., Papanikolau, A., van Gellicum-Bijlhout, J., van Oostenbruggen, J., Veugelers, D., Post-Uiterweer, A., . . Oosterlaan, J. (2009). Interference control in children with attention deficit/hyperactivity disorder. Journal of Abnormal Child Psychology, 37(2), 293-303. doi: https://doi.org/10.1007/s10802-008-9277-x

van Mourik, R., Sergeant, J. A., Heslenfeld, D., Konig, C., \& Oosterlaan, J. (2011). Auditory conflict processing in ADHD. Journal of Child Psychology and Psychiatry, 52(3), 265-274. doi: https://doi.org/10.1111/j.1469-7610.2010.02339.x

Vélez, A., Talero, C., González, R., \& Ibáñez, M. (2008). Prevalencia de trastorno por déficit de atención con hiperactividad en estudiantes de escuelas de Bogotá, Colombia. Acta neurológica colombiana, 24(1), 6-12. Recuperado de: https://www. acnweb.org/acta/2008_24_1_6.pdf 
Willcutt, E. G., Doyle, A. E., Nigg, J. T., Faraone, S. V., \& Pennington, B. F. (2005), Validity of the executive function theory of attention-deficit/hyperactivity disorder: a meta-analytic review. Biological Psychiatry, 57(11), 1336-1346. doi: https://doi.org/10.1016/j.biopsych.2005.02.006

Wolraich, M. L., McKeown, R. E., Visser, S. N., Bard, D., Cuffe, S., Neas, B., .. Danielson, M. (2014). The Prevalence of ADHD:Its Diagnosis and Treatment in Four School Districts Across Two States. Journal of Attention Disorders, 18(7), 563-575. doi: https://doi.org/10.1177/1087054712453169

Yang, B. R., Chan, R. C., Gracia, N., Cao, X. Y., Zou, X. B., Jing, J., . . Shum, D. (2011). $\mathrm{Cool}$ and hot executive functions in medication-naive attention deficit hyperactivity disorder children. Psychological Medicine, 41(12), 2593-2602. doi: https:// doi.org/10.1017/S0033291711000869

Yáñez-Téllez, G., Romero-Romero, H., Rivera-García, L., Prieto-Corona, B., Bernal-Hernández, J., Marosi-Holczberger, E., . . Silva-Pereyra, J. F. (2012). Cognitive and executive functions in ADHD. Actas Espanolas de Psiquiatria, 40(6), 293-298. Recuperado de: https://www.actaspsiquiatria.es/repositorio//14/80/ ENG/14-80-ENG-293-298-375974.pdf

Yordanova, J., Kolev, V., Albrecht, B., Uebel, H., Banaschewski, T., \& Rothenberger, A. (2011). May Posterror Performance Be a Critical Factor for Behavioral Deficits in Attention-Deficit/Hyperactivity Disorder? Biological Psychiatry, 70(3), 246-254. doi: https://doi.org/10.1016/j.biopsych.2011.02.026

Young, J. T., Carruthers, S., Kaye, S., Allsop, S., Gilsenan, J., Degenhardt, L., . . Preen, D. (2015). Comorbid attention deficit hyperactivity disorder and substance use disorder complexity and chronicity in treatment-seeking adults. Drug and Alcohol Review, 34(6), 683-693. doi: https://doi.org/10.1111/dar.12249 